\title{
Investigating the key factors in designing a communication skills program for medical students: A qualitative study
}

\author{
Seyyed M.Mahdi Hazavehei ${ }^{1}$, Hossein Karimi Moonaghi ${ }^{2}$, Babak Moeini ${ }^{3}$, Abbas Moghimbeigi ${ }^{4}$ Ali \\ Emadzadeh $^{5}$
}

${ }^{1}$ Ph.D. of Health Education and Promotion, Professor, Department of Public Health, School of Health, Hamadan University of Medical Sciences, Hamadan, Iran

${ }^{2}$ Ph.D. of Nursing Education, Associate Professor, Evidence-Based Caring Research Center, Department of Medical-Surgical Nursing, School of Nursing and Midwifery, Mashhad University of Medical Sciences, Mashhad, Iran

${ }^{3}$ Ph.D., Associate Professor, Social Determinants of Health Research Center \& department of public health , Hamadan University of Medical Sciences, Hamadan, Iran

${ }^{4} \mathrm{Ph}$.D. of Biostatistics, Associate Professor, Modeling of Non-communicable Disease Research Center, Department of Biostatistics, School of Health, Hamadan University of Medical Sciences, Hamadan, Iran

${ }^{5}$ Ph.D. Candidate of Health Education, Department of Public Health, Hamadan University of Medical Sciences, Hamadan, Iran

\section{Type of article: Original}

\begin{abstract}
Introduction: Medical students have a serious need to acquire communication skills with others. In many medical schools, special curriculums are developed to improve such skills. Effective training of communication skills requires expert curriculum design. The aim of this study was to explore the experiences and views of experts and stakeholders in order to design a suitable training program in communication skills for medical students.

Methods: The content analysis approach was used in this qualitative study. Forty-three participants were selected from the faculty, nurses, physicians, residents, and medical students at Mashhad University of Medical Sciences using purposive sampling. The data were collected through focus group discussions and semi-structured interviews. To ensure the accuracy of the data, the criteria of credibility, transferability, dependability, and conformability were met. The data were analyzed by MAXQDA software using the Graneheim \& Lundman model.

Results: The findings of this study consisted of two main themes, i.e., "The vast nature of the present communication skills training" and "administrative requirements of the training program regarding communication skills." The first theme included the educational needs of students, the problems associated with training people to have good communication skills, the importance of good communication skills in performing professional duties, communication skills and job requirements, the learning environment of communication skills, and the status of existing training programs for communication skills. Strategies and suitable methods for teaching communication skills and methods of evaluating the students in this regard also were obtained.

Conclusion: The findings of this study were the elements required to design a proper and local model to teach communication skills to medical students through analyzing the concepts of effective communication. The results of this study can be useful for medical faculties in designing a proper program for teaching medical students how to communicate effectively with patients and colleagues.

Keywords: communication skills, medical students, qualitative study, content analysis
\end{abstract}

\section{Corresponding author:}

Ali Emadzadeh, Department of Public Health, School of Health, Hamadan University of Medical Sciences, Hamadan, Iran. Tel: +98.5138942443, Email: emadzadeh92@gmail.com

Received: July 05, 2015, Accepted: September 01, 2015, Published: November 2015

iThenticate screening: September 01, 2015, English editing: September 27, 2015, Quality control: November 07, 2015

(C) 2015 The Authors. This is an open access article under the terms of the Creative Commons Attribution-NonCommercialNoDerivs License, which permits use and distribution in any medium, provided the original work is properly cited, the use is non-commercial and no modifications or adaptations are made. 


\section{Introduction}

Currently, communication skills and proper interpersonal communications are emphasized over and over in the healthcare delivery system. Good communication skills are very valuable and important for expressing emotions and needs and for reaching interpersonal goals. In other words, communication skills are a set of an individual's potential and actual abilities that, through proper use, result in behavior that can enhance awareness and contribute to attaining an acceptable level of emotional relationship with patients and colleagues alike. This behavior is called interpersonal communication skills (1). Undertaking effective communication to develop the relationship between doctors and patients and facilitating the teamwork with other health professionals is essential (2). Communication skills are teachable and learnable, and that is why medical schools throughout the world have implemented programs for teaching such skills (3). For instance, the Association of American Medical Colleges (AAMC) has emphasized the enhancement of communication skills as an important part of medical students' education (4). Medical students' ability to communicate and properly use communication skills is one of the most important factors that affect their learning quality and result in enhancing their performance in clinical environments. Thus, it is essential for medical students who will be members of the medical and health staff to master these skills. Fortunately, a significant part of medical students' training is conducted in clinical environments, which provides experience in conducting effective and targeted communications with the medical team, colleagues, and patients, resulting in improved communication skills (1). In many countries, in order to improve students' communication skills, instruction in this area is in integrated in the curriculum at various levels, including medical schools and residency. For instance, Dalhousie Canadian University has integrated these skills in the general medical curriculum as well as in graduate and continuing education programs (5). A review of the studies related to teaching communication skills to medical students and general practitioners indicated that instilling better communication skills through training has been successful worldwide. For instance, in one study, the performances of nine physicians regarding communication skills were evaluated after a training period using video and recorded analyses of interviews, and the evaluations indicated that the physicians who attended the training were more interested in and friendly toward their patients and colleagues and were applying more targeted skills (6). In general, it can be said that the necessity for teaching communication skills has been proven in many countries and there is a general agreement on the usefulness and importance of training medical students to have better communication skills. Of course, it should be noted that the effectiveness of teaching these skills requires proper, concise, and realistic planning of the appropriate training. Thus, it is important to use an appropriate process for designing the training to ensure that the training provides a framework for learning that is based on the professional requirements of the students (7). The World Health Organization (WHO) recommends that proper communications components should be determined based on the specific requirements of each culture and that the training to enhance communication skills should be set in accordance with these components (8). Conducting quantitative research alone would not be enough to accomplish this and to design an appropriate educational model. Thus, it also is necessary to use qualitative study to identify the important elements and structures needed to design and implement effective educational programs to enhance communication skills. This approach is important because it makes it possible to design and implement appropriate interventions to ensure that the objectives are met in this case. Therefore, since very little research has been conducted in Iran regarding the design of a suitable local model for teaching communication skills and since it is very important to learn the experiences and views of experts in this field, it is essential that such research be conducted. Thus, the main objective of this study was to explore the important factors involved in implementing an effective program for providing training designed to enhance the communication skills of medical students.

\section{Material and Methods}

\subsection{Study type}

This was a qualitative study, and it was conducted using the inductive content analysis method to characterize the diverse perspectives and experiences of participants in the study. This approach increases the understanding and perception of the data (9). In the inductive content analysis method, codes of data are extracted, and new concepts are related to the phenomena under study (10). In this study, the method of inductive content analysis was helpful in extracting the direct components from the acquired data.

\subsection{Participants and research environment}

In a qualitative study, samples include individuals who do not only have knowledge about or experience with the phenomena under study but are interested and willing to participate in the study. In a qualitative study, the sample size can vary, and the researcher continues the sampling process until new ideas are acquired (11). In the present study, data collection was conducted using purpose-based sampling, and it was continued until data saturation was 
reached. The 43 participants included members of the basic and clinical faculty, medical interns, nurses, and general practitioners and assistants working in healthcare centers. The research environment was Mashhad University of Medical Sciences, and the study was conducted between February and September 2014.

\subsection{Data collection}

The data in this study were collected through group interviews. The group interview approach was chosen since the subject under review was not confidential, and group discussion stimulated remembering and shaping views and experiences (12). The average meeting time was 95 minutes. The interviews were semi-structured, having open response questions. The group interviews were scheduled by two researchers (one with a Ph.D. degree in nursing education and one Ph.D. student in health education). The interview form consisted of two sets of questions, i.e., the main questions and the follow-up questions. The main questions were:

1) What is the status of teaching communication skills to medical students?

2) What communication skills do medical students need?

3) What strategies and approaches are appropriate for teaching communication skills to medical students in the existing conditions?

4) In the present circumstance, what methods are appropriate to evaluate these skills in medical students?

The researchers gained further insight into the participants' answers to each main question by proposing some follow-up questions, such as "Could you explain more?," "Can you continue this discussion by adding your own experience?," and "What do you mean when you say........?." All of the conversations were written down word for word.

\subsection{Trustworthiness}

Four criteria have been suggested to increase the precision and accuracy of qualitative research and the consolidation of the proposed data, i.e., credibility, transferability, dependability, and conformability (13). In this study, the researchers assigned adequate time to collect the data and tried to acquire real data through proper communication with the participants. Moreover, the member checking method was used to confirm the accuracy of the results and their conformity with the findings. Furthermore, to ensure the credibility of the data, the conversation codes that were used and the results that were obtained were submitted to three other researchers who were experts in health education and nursing and had professional experience in the subject of interest, and they expressed the same understanding as well. To check the consistency, which is equivalent to validity in quantitative research and, in fact, represents the stability of the data in the same time and conditions (14), random texts of conversations in the two group discussions were submitted to an external reviewer who was not involved with the research, and, for the most part, the same results were obtained. However, it should be noted that, in qualitative methods, unique experiences and perspectives are emphasized, and, even when all the conditions are created equally and similarly, similar results should not be expected (14). To ensure the generalizability of the data, we tried to choose participants in a way that maximized the diversity of features, such as gender, work experience, and age.

\subsection{Data analysis}

The data that were obtained were coded and analyzed using MAXQDA software, and the qualitative content analysis method was used to interpret the data that were entered into the software. In this method, codes and contents were recognized through systematic classification of the data. Content analysis goes beyond mere extraction of objective data by helping to uncover the hidden themes and patterns in the data (15). In this study, content analysis of the data was performed using the Graneheim \& Lundman approach. Hence, the implicit and explicit notions were coded and classified, and, then, the main themes were extracted from them according to the participants' descriptions. The codes were based on the semantic units derived from the participants' descriptions, and they were classified according to their similarities and differences.

\subsection{Ethical considerations}

Permission for this research was granted on December 5, 2014, by the Research Council of the University of Medical Sciences via Council No. 9309044310. In the interview sessions, first, the objective of the study was explained to the participants. Participation in this study was completely voluntary, and it was emphasized that the participants could withdraw from the study at any time. All participants were assured that their personal information would remain confidential and that all data would be destroyed after the study ended. Also, written permission for recording the conversations and interviews was obtained from each of the participants. 


\section{Results}

Given that the purpose of this study was to explore the experiences and views of experts and stakeholders to design a communication skills training program for medical students, two main themes were obtained, i.e., "the vast nature of the present communication skills training "and "administrative requirements of the training program regarding communication skills." The sub-theme of the study, i.e., "diversity in the present communication skills training status," consisted of several related factors, including the educational needs of students regarding communication skills; problems in training to enhance communication skills; the importance of communication skills in conducting professional duties; the importance of communication skills relative to job requirements; the learning environment of these skills; and the status of the educational program regarding such communication skills. The theme of "executive prerequisites of educational programs regarding communication skills" comprised appropriate training strategies, training methods used, and evaluation techniques of communication skills (Table 1).

Table 1. Themes and sub-themes regarding the communication skills of medical students

\begin{tabular}{|l|l|}
\hline Themes & Sub-themes \\
\hline $\begin{array}{l}\text { The vast nature of the present } \\
\text { communication skills training }\end{array}$ & $\begin{array}{l}\text { Educational needs of students regarding communication skills, difficulties } \\
\text { in training students to have better communication skills, the importance of } \\
\text { communication skills in performing professional duties, communication } \\
\text { skills and job requirements, educational environment, the status of the } \\
\text { existing educational program relative to teaching communication skills }\end{array}$ \\
\hline $\begin{array}{l}\text { Administrative requirements of } \\
\text { the training program regarding } \\
\text { communication skills }\end{array}$ & $\begin{array}{l}\text { Proper strategies for teaching communication skills, proper training } \\
\text { methods for teaching communication skills, methods of evaluating } \\
\text { students' communication skills }\end{array}$ \\
\hline
\end{tabular}

\subsection{The vast nature of the present communication skills training}

3.1.1. Educational needs of students

Medical students need to learn how to communicate effectively. Thus, the educational need can be defined as the gap that exits between the current status and the acceptable status in this regard. The findings of this study indicated that medical students' needs involve the three following issues:

3.1.1.1. Communication skills required

The analysis of the data indicated that medical students should learn to use precise verbal and nonverbal communication skills. These skills include various items, such as the proper distance from the audience, expressing empathy, active listening, persuading, increasing the listener's confidence, how to give bad news to patients and their companions, effective communication methods, and a good appearance.

3.1.1.2. The ability to communicate with different audiences

Based on the results, medical students, due to the requirements of their jobs, must communicate with various groups, such as lecturers, assistants, patients and their companions, and even society in general. In this regard, one of the participants stated that "medical students should learn how to communicate with classmates, lecturers, patients, and their companions as well as the personnel and all the other people."

3.1.1.3. The need to be familiar with cultural and social roles in conducting effective communication

The findings of this study indicated that social skills and sociology, cultural issues, cultural differences are the important factors that medical students should be aware of and treat with sensitivity. Most of the participants who belonged to different groups in this study emphasized that the effect of cultural and socioeconomic issues should be incorporated in the training provided to medical students concerning communication skills. One of the medical practitioners who participated in the study stated that "based on my experience, I have realized that I should observe some cross lines and cultural issues while communicating with patients."

3.1.2. Existing problems

Based on the views and experiences of the participants, some problems have resulted from the inadequate attention that is given to teaching medical students about the importance of good communication skills. The data in this regard indicated that there were many problems, including incorrect views toward patients and disregard of their human dimension, emphasis on resident-centered learning, students' passiveness, being worried about job security in the future, theoretical learning of communication skills, and limited social interactions of students prior to entering the university. Regarding the latter issue, one of the participants emphasized that "medical students have such limited social interactions due to studying a lot and this has affected negatively on their communication method." 


\subsubsection{Learning limitations}

According to the participants' experiences, some limitations have made the learning of communication skills difficult. Our findings showed that some of these limitations are personal and depend of the students themselves. One of the participants (a nurse) mentioned that "some students have a sense of superiority and selfishness and consider themselves as being a doctor and results in not making a good interaction." Among the other limitations that are considered personal are a lack of interest, anxiety, feelings of inadequacy, a lack of interest in medical science, and conflicts. However, some other limitations are not related to the students and are not considered personal. Some examples of such limitations are the communication models observed by students, such as bad communication of professors and residents with patients and others.

3.1.4. The need to learn good communication skills to perform professional duties

The findings showed that proper use of communication skills results in better performance of students in terms of her or his professional duties. One of the participants emphasized that "if we look at the graduates we find that those who have stronger communication skills are more at least 15 to 20 percent more successful compared with others." Issues such as considering the identity of the audience as a human, considering the patients' and their companions' rights, and obeying the morality are professional duties of medical students that will be performed better if communication skills are learned.

3.1.5. The role of communication skills in job success

Results of the data analysis in this study showed that learning proper communication skills could have a positive effect on students' job success as a doctor. One of the participants (a practitioner) stated that "patients should be treated in a way that they become consent while they are with us, for instance we should listen to them attentively," and another participant (an assistant) purported that "some doctors who are highly educated do not have many patients and that is because they do not have good communication skills."

3.1.6. Characteristics of the target group

Experiences of the participants represented that diverse characteristics of medical students are important in their learning communication skills and in the effectiveness of training they are offered. According to the information received from the participants, students who had better emotional intelligence and more experience and had better family upbringing were learning and implementing better communication skills. A participant (nurse) stated in this regard that "some students are very good in regards to family and cultural upbringing and know communication and respecting well and mostly make a better contact with the clinical staff and patients."

3.1.7. Educational environment

The problems that exist in the educational environment result in the students' inability to learn, practice, and implement good communication skills. Some of the issues that were identified in this educational environment were a lack of discipline in clinical departments, lack of facilities for students in the departments, the large numbers of students, the faculty's lack of time, and the long duty hours of the students. Most of the participants emphasized that the environment and proper facilities, especially in clinical departments, could facilitate the process of training professionals to have good communication skills.

3.1.8. Existing educational programs

Our analysis of the data indicated that there was inadequate emphasis on the importance of good communication skills in the general clinical training program. The findings of this study indicated that the following three important issues in the educational program.

3.1.8.1. Lack of clear expectations of the students regarding their communication skills

Unclear duties of students in departments and lack of clear expectations of students regarding their communication skills have made the learning of these skills problematic.

3.1.8.2. Lack of a clear model for training students to have good communication skills

According to the participants' experiences, applying a clear model and following it in the educational process would have a great impact on the students' learning communication skills. The analysis of the data in this research showed that the training provided to enhance communication skills currently is not performed using a clear and planned model, so it is not very effective. In this regard, one of the participants emphasized the point that "I have concluded that having a model is not important in training, especially training the communication skills. I have asked some people questions regarding how they teach such skills, what contents do you present, how many people should be present in the sessions? And I concluded that they do not follow a special model in this regard." According to the findings of this study, it is necessary to categorize the details of the students' required skills separately for each department for which they receive training.

3.1.9. Inappropriateness of the training programs

The findings showed that insufficient attention was being given to teaching communication skills, and there were several related issues, including a lack of attention to teamwork and team-based learning, insufficient transfer of 
knowledge regarding communication skills, lack of course units, inadequate attention to the scientific basis in designing the training to enhance communication skills. These items were identified as contributing to the insufficiency of the existing programs for improving the communication skills of medical students. Regarding the educational program, one of the participants stated that "During the education years, no trainings were conducted on vital and important subjects, such as communication skills that doctors truly require."

\subsection{Executive requirements of the training program of communication skills}

\subsubsection{Educational strategy}

One of the important points in training medical students with respect to their communication skills is to apply proper and applicable strategies. Based on the analysis of the data acquired in this study, five strategies were identified.

3.2.1.1. Training through others

The findings showed that one of the appropriate approaches to teaching communication skills to medical students is to use medical lecturers, nurses, and their professional assistants to supplement the training of these skills by their own lecturers. In addition, professionals in other fields could be involved in informal training. Concerning this issue, one of the participants stated that "we could use associations and student organizations and communities for training the communication skills to medical students."

\subsubsection{Community-based training}

According to this strategy, training students to have good communication skills should be in accordance with the requirements of the healthcare system and through contacts with various cultures. Knowing the society's culture is important for students to learn appropriate communication skills, therefore, it is suggested that a local model be designed and used in teaching these skills in medical schools.

3.2.1.3. Continuous training

Analysis of the data showed that, although it is necessary to consider a clear program for teaching communication skills to medical students at each level of their education, the general strategy of providing training in appropriate communications should be emphasized from enrollment to graduation.

3.2.1.4. Training in the real environment

Most of the participants in this research emphasized that training medical students to have better communication skills is more effective in their real environments because that setting facilitates the students' learning of these skills. 3.2.1.5. Virtual training and training in a simulated environment

Our analysis of the data showed that virtual training, especially when simulated patients and simulation software are used, is an appropriate strategy for training medical students to have good communication skills.

3.2.2. Training methods

Communication skills should be used in a ways that allow more practice and involvement of students in the learning process. The participants in this study emphasized various teaching methods and techniques, including role-playing, using video clips, independent learning, using modern technology, and using social networking, workshops, practical training, and multimedia programs. In general, training methods that are interesting to the students and result in more involvement in the learning process are more effective ways of teaching communication skills. Due to this, according to one participant "faculties' working experience that is gained over years is a useful resource for learning communication skills by medical students, and it should be used in education." Using faculties' experiences also could be an appropriate method to teach communication skills to students.

3.2.3. Evaluation of the communication skills of medical students

The analysis of the data identified the following four sub-themes during the evaluation of communication skills.

3.2.3.1. Existing problems regarding evaluation

The research findings indicated that, despite the importance of medical students' learning communication skills, their communication skills are not consider in their evaluations and no scores are allocated to them.

3.2.3.2. Evaluation strategies

Continuous evaluation of communication skills should be conducted at various levels of study, especially in clinical departments. Appropriate strategies that could be used to evaluate medical students' communication skills include the use of focused tests, such as pre-internship tests and asking other members of health team to take part in the process of observing and evaluating medical students' communication skills.

\subsubsection{Evaluation method}

The participants in this study emphasized some evaluation methods for assessing the communication skills of medical students, including direct observation; self-assessment based on evidence, portfolios, and work-based assessments; and using the 360-degree test. 


\subsubsection{Providing feedback to students}

Providing feedback is an integral part of training students to have better communication skills. In this regard, one of participants (a nurse) emphasized that "those who are involved with teaching the communication skills to students should analyze their behaviors in this regard." The analysis of the data also showed that presenting frequent and individual feedback results in the improvement of the students' communication skills.

\section{Discussion}

The findings of this study facilitated a better understanding of the required elements to design a proper and local model for teaching communication skills to medical students. The sub-themes of the students' educational needs with respect to communication skills were consistent with the results of a 2012 study conducted by Barati et al. (16). Their study revealed that communication skills of medical students were unfavorable at all verbal, auditory, and feedback levels and that the students must be taught better communication skills, which would require experimental training of communication skills. When the London Health Department defined the expected communication skills for health-related jobs, the understanding of cultural differences was mentioned and emphasized (17). This is consistent with one of the sub-themes of our study, i.e., "the need to become familiar with cultural and social roles in conducting effective communication." Berkhof et al. conducted a systematic review to determine the effective educational strategies for training physicians to have and use appropriate communication skills, and they stated that that educational programs designed to teach communication skills should include active, performance-based strategies. Their results showed that programs that were student-centered and emphasized the continuous practice of skills were more successful in teaching communication skills (18). The findings of our study in the sub-theme "Executive requirements of the training program of communication skills" indicated that the training strategy should be implemented in a real environment and that students who are involved in the training process should receive practical training and feedback concerning their status, including areas in which improvements should be made. Holding workshops to train students to have better communication skills was consistent with the recommendation of a study conducted by Attari Moghaddam et al. (19). Their study showed that physician-patient workshop training for medical students was very effective in improving the students' knowledge and understanding concerning the importance of good communications. As using direct observation and self-assessment based on evidence were emphasized as proper methods in the evaluation method of communication skills in our study, Lee \& Lee also aimed to evaluate the effectiveness of a communication skills training program for medical students prior to enter the clinical stage. In addition, they used the self-assessment and observation to evaluate the communication skills of students prior to and after attending the mentioned educational sessions. As indicated by the results of their study, students communication skills score showed a significant increase with both methods (20).

\section{Conclusions}

As indicated by the results of the study, in order to develop an appropriate training program for medical students concerning their communication skills, two main themes should be considered, i.e., diversity in the present communication skills training status and the administrative requirements of the training program. To enhance the communication skills of medical students, localized programs for this purpose should be planned and implemented. Therefore, it is recommended that medical schools provide training in communication skills for their students based on their own conditions with emphasis on training in the clinical settings. Further research should be conducted on quantitative, interventional studies with the aim of developing training programs and assessing their effects on the communication capabilities of medical students.

\section{Acknowledgments:}

This article is part of a Ph.D. thesis in health education with code 9309044310 that was approved by Hamadan University of Medical Sciences. The authors sincerely thank all participants in this research, including those who contributed to the research, including the scientific board, students, residents, doctors, and nurses at Mashhad University of Medical Sciences. This research would not have been possible without their cooperation and assistance.

\section{Conflict of Interest:}

There is no conflict of interest to be declared.

Authors' contributions:

All authors contributed to this project and article equally. All authors read and approved the final manuscript. 


\section{References}

1) Salimi M, Peyman H, Sadeghifar J, Toloui Rakhshan S, Alizadeh M, Yamani N. Assessment of Interpersonal Communication Skills and Associated Factors among Students of Allied Medicine School in Tehran University of Medical Sciences. Iranian Journal of Medical Education. 2013; 12(12): 895-902.

2) Conn JJ, Lake FR, McColl GJ, Bilszta J, and Woodward-Kron R. Clinical teaching and learning: from theory and research to application. Med J Aust. 2012; 196(8):527. doi: 10.5694/mja10.11473, PMid: 22571313

3) Soltani Arabshahi SK, Ajami A, Siabani S. Investigation of Doctor-Patient Communication Skills Teaching: Medical Learners' Perception (Stager-Intern) and Staffs of Iran University of Medical Sciences \& Kermanshah University of Medical Sciences. Journal of Iran University of Medical Sciences. 2002; 11(41): 423-33.

4) Wright KB, Bylund C, Ware J, Parker P, Query JL, Baile W. Medical student attitudes toward communication skills training and knowledge of appropriate provider-patient communication: a comparison of first-year and fourth-year medical students. Medical Education Online. 2009; 11.

5) Communication skills program. Dalhousie University;2013; Available from: http://csp.medicine.dal.ca/cross-Training.php

6) Mirzazadeh A, Jahanian I, Shahi F, JafariSirous KE. Comparative study of opinions of patients and clinical faculty members of Babol University of medical sciences on patient-centeredness in doctor-patient relationship using patient-practitioner orientation scale. Journal of Babol University of Medical Sciences. 2010.

7) Thorndyke LE, Gusic ME, George JH, Quillen DA, Milner RJ. Empowering junior faculty: Penn State's faculty development and mentoring program. Academic medicine. 2006; 81(7): 668-73. doi: 10.1097/01.ACM.0000232424.88922.df. PMid: 16799296

8) Zamani A, Shams B, Moazzam E. Communication Skills Training for Physicians as a Strategy for Enhancing Patients' Satisfaction: A Model for Continuing Education. Iranian Journal of Medical Education. 2004; 4(1): 15-22.

9) Elo S, Kyngäs H. The qualitative content analysis process. J Adv Nurs. 2008; 62(1): 107-15. doi: 10.1111/j.1365-2648.2007.04569.x, PMid: 18352969

10) Moretti F, van Vliet L, Bensing J, Deledda G, Mazzi M, Rimondini M, et al. A standardized approach to qualitative content analysis of focus group discussions from different countries. Patient Educ Couns. 2011; 82(3): 420-8. doi: 10.1016/j.pec.2011.01.005, PMid: 21292424

11) Polit DF, Beck CT. Essentials of nursing research: Appraising evidence for nursing practice: Lippincott Williams \& Wilkins; 2013.

12) KarimiMoonaghi H DA, Khajedalouei M, DashtiRahmatabadi M, Binaghi T. Lived clinical learning experiences of medical students. Iranian Journal of Medical Education. 2012; 11(6).

13) Denzin NK, Lincoln YS. The Sage handbook of qualitative research: Sage; 2005.

14) AdibHajbagheri M PS, Salsali M. Methods of Qualitative Research. Tehran: Boshra; 2010.

15) Hsieh H-F, Shannon SE. Three approaches to qualitative content analysis. Qual Health Res. 2005; 15(9): 1277-88. doi: 10.1177/1049732305276687, PMid: 16204405

16) Barati M, Moeini B, Samavati A, Salehi O. Assessment of communication skills level among medical college students: verbal, listening, and feedback skills. Journal of Nursing and Midwifery of Urmia University of Medical Sciences. 2012; 10(2).

17) Communication skills education for doctors. The board of medical education of the British Medical Association, 2004.

18) Berkhof M, van Rijssen HJ, Schellart AJ, Anema JR, van der Beek AJ. Effective training strategies for teaching communication skills to physicians: an overview of systematic reviews. Patient Educ Couns. 2011; 84(2): 152-62. doi: 10.1016/j.pec.2010.06.010, PMid: 20673620

19) AttariMoghadam J, Mokhlespour S, Valizadeh M, Momtazi S, Sharifi F, Ghodrati S, et al. Teaching"Doctor-Patient Relationship"to the Medical Student: Assesment of Knowledge and Satisfaction. Journal of Medical Education Development. 2010; 3(4): 26-33.

20) Lee YM, Lee YH. Evaluating the short-term effects of a communication skills program for preclinical medical students. Korean J Med Educ. 2014; 26(3): 179-87. doi: 10.3946/kjme.2014.26.3.179, PMid: 25805260 